PROCEEDINGS OF THE

AMERICAN MATHEMATICAL SOCIETY

Volume 129, Number 9, Pages 2709-2713

S 0002-9939(01)05859-2

Article electronically published on February 9, 2001

\title{
A CHARACTERIZATION OF HEAT BALLS BY A MEAN VALUE PROPERTY FOR TEMPERATURES
}

\author{
NORIAKI SUZUKI AND NEIL A. WATSON
}

(Communicated by Albert Baernstein II)

\begin{abstract}
We discuss an inverse mean value property of solutions of the heat equation. We show that, under certain conditions, a volume mean value identity characterizes heat balls.
\end{abstract}

\section{Introduction}

There are many papers that deal with the inverse mean value property for harmonic functions (see [3] for an excellent survey). Above all, Ü.Kuran 2] gave a simple and elegant proof of the following result, which means that harmonic functions characterize a ball: Let $D$ be an open set in $n$-dimensional Euclidean space, with finite volume $|D|$ and containing the origin 0 . If

$$
h(0)=\frac{1}{|D|} \int_{D} h(x) d x
$$

holds for every integrable harmonic function $h$ on $D$, then $D$ is a ball centered at the origin. Note that the boundary of a ball is a level surface of the Newtonian kernel (or logarithmic kernel if $n=2$ ). Similar mean value results for heat operator are less well-known. In this paper we will give a result on the characterization of heat balls - which are level surfaces of the Gauss-Weierstrass kernel - by a mean value property for temperatrures.

For any point $P$ in $(n+1)$-dimensional Euclidean space $\mathbf{R}^{n+1}$, we write $P=$ $(x, t)=\left(x_{1}, \ldots, x_{n}, t\right)$. Let $H$ denote the heat operator and $H^{*}$ its adjoint; that is,

$$
H=\sum_{i=1}^{n} \frac{\partial^{2}}{\partial x_{i}^{2}}-\frac{\partial}{\partial t} \quad \text { and } \quad H^{*}=\sum_{i=1}^{n} \frac{\partial^{2}}{\partial x_{i}^{2}}+\frac{\partial}{\partial t} .
$$

We use $W$ to denote the Gauss-Weierstrass kernel, defined by

$$
W(x, t)= \begin{cases}(4 \pi t)^{-n / 2} \exp \left(-\|x\|^{2} / 4 t\right) & \text { if } t>0, \\ 0 & \text { if } t \leq 0,\end{cases}
$$

where $\|x\|=\left(x_{1}^{2}+\cdots+x_{n}^{2}\right)^{1 / 2}$.

Received by the editors July 28, 1999 and, in revised form, January 20, 2000.

2000 Mathematics Subject Classification. Primary 31B10, 35K05.

Key words and phrases. Heat ball, temperature, supertemperature, mean value property, Gauss-Weierstrass kernel. 
Let $P_{0}=\left(x_{0}, t_{0}\right) \in \mathbf{R}^{n+1}$ and $c>0$. We shall use two kinds of ball with centre $P_{0}$ and radius $c$. The first is the usual open ball

$$
B\left(P_{0}, c\right)=\left\{(x, t) \in \mathbf{R}^{n+1}:\left\|x-x_{0}\right\|^{2}+\left(t-t_{0}\right)^{2}<c^{2}\right\},
$$

and the second is the heat ball

$$
\Omega\left(P_{0}, c\right)=\left\{(x, t) \in \mathbf{R}^{n+1}: W\left(x_{0}-x, t_{0}-t\right)>(4 \pi c)^{-n / 2}\right\} .
$$

It is easy to check that $\Omega\left(P_{0}, c\right)$ is a convex subdomain of the cylinder $\{(x, t) \in$ $\left.\mathbf{R}^{n+1}:\left\|x_{0}-x\right\|^{2}<2 n c / e, t_{0}-c<t<t_{0}\right\}$.

Mean value theorems for temperatures that involved integrals over heat balls were established by Pini [4] (see also [8]) and Watson [6]. Here we consider the latter form as the kernel is simpler, but the method works for either and the same result holds for the Pini kernel. More precisely, we will prove the following inverse mean value property. Here, and subsequently, $\chi_{A}$ denotes the characteristic function of a set $A$ in $\mathbf{R}^{n+1}$.

Theorem. Let $c>0$, and let $D$ be a bounded open set in $\mathbf{R}^{n+1}$. If the following conditions are satisfied, then $D=\Omega(0, c)$.

(i) The function

$$
f(x, t):=\left(\chi_{D}(x, t)-\chi_{\Omega(0, c)}(x, t)\right) \frac{\|x\|^{2}}{t^{2}}
$$

belongs to $L^{p}\left(\mathbf{R}^{n+1}\right)$ for some $p>(n / 2)+1$.

(ii) For all $(y, s) \in \mathbf{R}^{n+1} \backslash D$,

$$
\iint_{D} W(x-y, t-s) \frac{\|x\|^{2}}{t^{2}} d x d t=2^{n+2}(\pi c)^{n / 2} W(y,-s) .
$$

Conditions (i) and (ii) require some explanation. In the harmonic case, the condition that corresponds to (i) is that $0 \in D$, which means that $D$ is indistinguishable from the appropriate ball in the vicinity of 0 . The direct analogue in the present case is that $D$ is indistinguishable from $\Omega(0, c)$ in the vicinity of 0 , and condition (i) is a generalization of that. In the harmonic case, although Kuran assumed (1) for all integrable harmonic functions, he actually used it only for certain Poisson kernels (and constants). The direct analogue of (ii) was later used by Aharonov, Schiffer and Zalcman in [1] (along with the essence of Kuran's argument) to prove another characterization of balls by harmonic functions.

\section{Proof of the theorem}

Due to the fact that the centre of a heat ball lies on the boundary, many methods that work for the harmonic case cannot be adapted to the heat equation. Our proof is based on an argument in [5, p.30]. The necessary potential theory can be found in [7].

Put $\Omega=\Omega(0, c)$ and $\alpha=2^{n+2}(\pi c)^{n / 2}$. By the volume mean value property of temperatures in [6, p.409], we have $\iint_{\Omega}\|x\|^{2} / t^{2} d x d t=\alpha$ and

$$
\iint_{\Omega} W(x-y, t-s) \frac{\|x\|^{2}}{t^{2}} d x d t=\alpha W(y,-s) \quad \forall(y, s) \in \mathbf{R}^{n+1} \backslash \Omega .
$$


Since $D$ is bounded, there is $s<0$ such that $D \cap\left(\mathbf{R}^{n} \times\{s\}\right)=\emptyset$, so that

$$
\begin{aligned}
\alpha & =\alpha \int_{\mathbf{R}^{n}} W(y,-s) d y \\
& =\int_{\mathbf{R}^{n}}\left(\iint_{D} W(x-y, t-s) \frac{\|x\|^{2}}{t^{2}} d x d t\right) d y \\
& =\iint_{D} \frac{\|x\|^{2}}{t^{2}} d x d t,
\end{aligned}
$$

and hence

$$
\iint_{D} \frac{\|x\|^{2}}{t^{2}} d x d t=\iint_{\Omega} \frac{\|x\|^{2}}{t^{2}} d x d t
$$

Now for $(y, s) \in \mathbf{R}^{n+1}$, we put

$$
v(y, s)=\iint_{D} W(x-y, t-s) \frac{\|x\|^{2}}{t^{2}} d x d t,
$$

$v_{0}(y, s)$ equal to the corresponding integral over $\Omega$,

$$
u(y, s)=\alpha W(y,-s)-v(y, s)
$$

and

$$
u_{0}(y, s)=\alpha W(y,-s)-v_{0}(y, s) .
$$

Then (ii) implies that

$$
u(y, s)=0 \quad \forall(y, s) \in \mathbf{R}^{n+1} \backslash D,
$$

and (2) implies that

$$
u_{0}(y, s)=0 \quad \forall(y, s) \in \mathbf{R}^{n+1} \backslash \Omega .
$$

In [6], the volume mean value theorem was established by integrating the corresponding surface mean. By the example in [8], for each $(y, s)$ the surface mean of $W(x-y, t-s)$ over $\partial \Omega$ is $(4 \pi c)^{-n / 2} \wedge W(y,-s)$. Therefore, for all $(y, s) \in \Omega$ we have

$$
\begin{aligned}
u_{0}(y, s) & =\alpha W(y,-s)-2^{n+1} \pi^{n / 2} n \int_{0}^{c} \ell^{n / 2-1}\left((4 \pi \ell)^{-n / 2} \wedge W(y,-s)\right) d \ell \\
& >\alpha W(y,-s)-2^{n+1} \pi^{n / 2} n \int_{0}^{c} \ell^{n / 2-1} W(y,-s) d \ell \\
& =0 .
\end{aligned}
$$

We assert that $v-v_{0} \in C\left(\mathbf{R}^{n+1}\right)$. Let $f$ be the function defined in (i). Observe that condition (ii), with $(y, s)$ chosen so that $s=0$, implies that $\operatorname{supp}(f) \subseteq \mathbf{R}^{n} \times$ $(-\infty, 0]$. For each $a \leq 0$, let $f_{a}$ denote the restriction of $f$ to $\mathbf{R}^{n} \times(-\infty, a)$, and let $K_{a}=\operatorname{supp}(f) \cap\left(\mathbf{R}^{n} \times[a, 0]\right)$. If $a<0$, then $f_{a}$ is bounded, so that the function

$$
(y, s) \mapsto \iint_{\mathbf{R}^{n+1}} W(x-y, t-s) f_{a}(x, t) d x d t
$$

is continuous. Since

$$
H^{*}\left(\iint_{K_{a}} W(x-y, t-s) f(x, t) d x d t\right)=0 \quad \forall(y, s) \in \mathbf{R}^{n+1} \backslash K_{a},
$$


it follows that $v-v_{0} \in C\left(\mathbf{R}^{n+1} \backslash K_{a}\right)$. Since $a$ is arbitrary, $v-v_{0} \in C\left(\mathbf{R}^{n+1} \backslash K_{0}\right)$. Finally, if $q:=p /(p-1)$, the exponent conjugate to $p$, then $q<(n+2) / n$ and for some constant $M$ we have

$$
\left|v-v_{0}\right|(y, s) \leq M|s|^{(n+2-n q) / 2 q}\|f\|_{p},
$$

so that condition (i) implies that $\left(v-v_{0}\right)(y, s) \rightarrow 0$ as $s \rightarrow 0$.

To prove that $D=\Omega$, it is sufficient to show that $\chi_{D}=\chi_{\Omega}$ a.e. on $\mathbf{R}^{n+1}$. For then $u=u_{0}$, so that (4) and (6) imply that $\Omega \subseteq D$. Therefore $\Omega=D \backslash K$ for some relatively closed subset $K$ of $D$ with measure zero. Since $\bar{\Omega}^{o}=\Omega$, it follows that $D=\Omega$.

Suppose that $\chi_{D} \neq \chi_{\Omega}$ on a set of positive measure. Since $\chi_{\bar{\Omega}}=\chi_{\Omega}$ a.e., we can choose $P_{0} \in D \backslash \bar{\Omega}$, in view of (3). If $L$ is any line through $P_{0}$, we can choose $Q_{1}, Q_{2} \in L \cap \partial D$ such that $P_{0}$ belongs to the segment $Q_{1} Q_{2}$. If $Q_{1}$ and $Q_{2}$ both belonged to $\bar{\Omega}$, then by convexity $P_{0}$ would also belong to $\bar{\Omega}$, which is false. Therefore $\partial D \backslash \bar{\Omega} \neq \emptyset$. Moreover, $\partial D \backslash \bar{\Omega}$ contains a point $\left(y_{0}, s_{0}\right)$ with the property that every ball centred there meets $D_{+}=D \cap\left(\mathbf{R}^{n} \times\left(s_{0}, \infty\right)\right)$. For otherwise $\partial D \backslash \bar{\Omega}$ would be contained in the union of a sequence of parallel hyperplanes, and so $D$ would be unbounded. Choose a ball $B$, centred at $\left(y_{0}, s_{0}\right)$, such that $B \cap \bar{\Omega}=\emptyset$. The function $u$ is an $H^{*}$-subtemperature on $B$, is not an $H^{*}$-temperature on $B \cap D$, and is zero at $\left(y_{0}, s_{0}\right)$ by (4). Since $B \cap D_{+} \neq \emptyset$, the maximum principle therefore implies that

$$
\sup _{B} u>0
$$

Put

$$
m=\max _{\mathbf{R}^{n+1}}\left(u-u_{0}\right) \quad \text { and } \quad E=\left(u-u_{0}\right)^{-1}(m) .
$$

Since $B \cap \bar{\Omega}=\emptyset$, we have $u_{0}=0$ on $B$. Therefore $\sup _{B}\left(u-u_{0}\right)>0$, and hence $m>0$. Because $u_{0} \geq 0$ by (5) and (6), we have $u>0$ on $E$, and hence $E \subseteq D$ by (4). On the other hand, for all $(x, t) \in D$ we have

$$
H^{*}\left(u-u_{0}\right)(x, t)=\left(1-\chi_{\Omega}(x, t)\right) \frac{\|x\|^{2}}{t^{2}} \geq 0,
$$

so that $u-u_{0}$ is an $H^{*}$-subtemperature on $D$. The maximum principle now implies that $E \cap \partial D \neq \emptyset$, a contradiction. Hence $D=\Omega$.

\section{REFERENCES}

[1] D. Aharonov, M.M. Schiffer and L. Zalcman, 'Potato kugel', Israel J. Math., 40(1981), 331339. MR 83d:31002

[2] Ü. Kuran, 'On the mean-value property of harmonic functions', Bull London Math. Soc., 4(1972), 311-312. MR 47:8887

[3] I. Netuka and J. Veselý, 'Mean value property and harmonic functions', Classical and modern potential theory and applications (eds. K. Gowrisankaran et al), Kluwer, Dordrecht (1994), 359-398. MR 96c:31001

[4] B. Pini, 'Maggioranti e minoranti delle soluzioni delle equazioni paraboliche', Ann. Mat. Pura Appl., 37(1954)249-264. MR 16:593f

[5] H.S. Shapiro, The Schwarz function and its generalization to higher dimensions, John Wiley \& Sons, New York, 1992. MR 93g:30059

[6] N.A. Watson, 'A theory of subtemperatures in several variables', Proc. London Math. Soc., 26(1973)385-417. MR 47:3838 
[7] N.A. Watson, 'Green functions, potentials, and the Dirichlet problem for the heat equation', Proc. London Math. Soc., 33(1976)251-298; Corrigendum, ibid., 37(1978)32-34. MR 58:17149

[8] N.A. Watson, 'A convexity theorem for local mean values of subtemperatures', Bull. London Math. Soc., 22(1990)245-252. MR 91b:31012

Graduate School of Mathematics, Nagoya University, Nagoya, 464-8602, Japan

E-mail address: nsuzuki@math.nagoya-u.ac.jp

Department of Mathematics and Statistics, University of Canterbury, Christchurch, New Zealand

E-mail address: naw@math.canterbury.ac.nz 